\title{
The Properties of the Cornea Based on Hyperspectral Imaging: Optical Biomedical Engineering Perspective
}

\author{
S.S.M.Noor ${ }^{1}$, K.Michael ${ }^{2}$ S.Marshall $^{1}$, J.Ren ${ }^{1}$, J.Tschannerl ${ }^{1}$, F.J.Kao ${ }^{3}$ \\ ${ }^{1}$ Centre of Excellent Signal and Image Processing, E.E.E, University of Strathclyde,Glasgow, Scotland, United Kingdom. \\ ${ }^{2}$ Glasgow Centre for Ophthalmic Research, Gartnavel General Hospital, Glasgow, Scotland, United Kingdom. \\ ${ }^{3}$ Institute of Biophotonics, National Yang-Ming University, Taipei, Taiwan.
}

siti-salwa-binti-md-noor@strath.ac.uk

\begin{abstract}
Biomedical engineering is a unique area that allows fusion between two distinct fields of engineering and medicine. The integration of efforts from both fields promises progress through acquisition of information from tissues, cells, and organs through non-invasive methods of assessment. Here we investigate the ability of a hyperspectral device in extracting data from tissues through the wavelength spectrum, in foreseeing its potential in clinical diagnostics by simplifying methods of examination by clinicians in detecting corneal injuries. Hyperspectral imaging using 400 to $1000 \mathrm{~nm}$ visible wavelength was used to scan five porcine eyes (injured and non-injured). Images were saved in three dimensional images of rows, columns, and depth slices at 1200 to $1300 \times 804 \times 604$ and were processed. All laboratory works were performed in accordance with the general risk assessment of University of Strathclyde. In our results, analysis of the images reveals significant cue between 500 to $800 \mathrm{~nm}$ bands in differentiating between injured and noninjured parts of the eye.
\end{abstract}

Keywords - cornea epithelium, spectral imaging, biomedical engineering

\section{INTRODUCTION}

The human cornea [2] consists of 5 distinct layers, namely the Epithelium, Bowman membrane, Stroma, Descemet membrane, and Endothelium. The purpose of this paper is to discuss the potential for hyperspectral imaging to measure the properties of the outermost layer of the cornea which is the epithelium. When injured, epithelial cells have an innate ability to self-heal which depends on the level and depth of injury. The common methods used by an eye specialist to investigate cornea injuries include slit lamp [3], confocal microscopy [4], optical coherence tomography $[5,6]$ (OCT), or corneal staining [7]. These methods while useful present some challenges in the real life setting. They are costly, involving usually heavy and complex machinery and often require a specialist operator.

Hence, we propose a noninvasive system which we envisage will overcome the above challenges and assist clinicians in quantifying corneal epithelial injuries in a more objective manner. The overall system is summarised in Fig.1. The block diagram comprises of four main components; image acquisition through the capturing and processing of an image, a spectral device to provide spatial and spectral signatures of reflectance, a subject to be observed (here we refer to cornea on a porcine eye), and finally a light source to ensure sufficient radiance is present at the subject. Further details are discussed in the following topics.

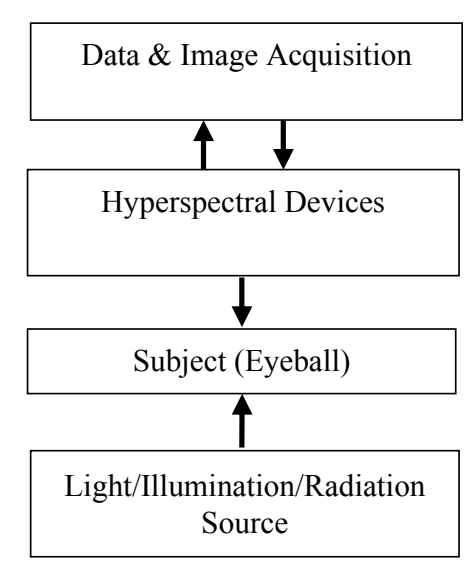

Figure 1.System block diagram.

\section{RESEARCH BACKGROUND}

\section{A. Corneal Histology}

The cornea is a transparent stratum on the eyeball. Its translucent property allows light to enter the eye. The Cornea is comprised of three essential layers namely; epithelium on the outermost layer, stroma in the middle, and endothelium as the innermost layer. The membrane separating the epithelium and stroma is known as the Bowman membrane and the Descemet membrane lies between the stroma and endothelium. The role of the cornea can be summarised succinctly: Protection, transmission and refraction [8].

The corneal epithelium is a tight, protective, stratified squamous epithelium which typically comprises 5-7 layers of cells and is firmly attached to the underlying stroma [8]. The outermost cells are approximately $50 \mu \mathrm{m}$ in width [2]. The epithelium protects the underlying layers and is therefore often subjected to external contact, causing it to be a common site of injury on the cornea.

The stroma constitutes ninety percent of the overall corneal thickness. It appears as organized structured, dense, avascular and relatively acellular connective tissue comprising collagen and proteoglycans. The corneal endothelium is a 4-6 $\mu \mathrm{m}$ thick monolayer of approximately 400,000 cells arranged in a hexagonal mosaic [8]. This layer is essential in keeping the cornea clear as it helps pump water out of this transparent structure. 
Corneal damage can occur at different levels, and often injuries are confined to the superficial epithelial layer. Such injuries trigger several mechanisms that contribute to the healing process which includes wound size, depth, causative agents and the tear quality [9]. Following prolonged epithelial injury, the deeper stromal surface is exposed and becomes irregular, causing the stroma to swell and cloud. Ulceration and scarring may follow causing the eye to become vulnerable to infection. Repetitive assault to the cornea either in acute or chronic disease is responsible for reduction of vision in a large number of people worldwide [2].

Efforts to objectively quantify epithelial cell damage are still scant in the current literature. At present the most commonly used method to assess corneal epithelial damage is carried out by a Dye test (fluorescein, rose Bengal, lissamine green) with the use of a slit lamp machine.[10] This method allows the clinician to assess the extent of damage although often without any robust scale or objective measures.

\section{B. Medical Spectral Imaging}

Hyperspectral Imaging (HSI) is a hybrid modality that combines imaging and spectroscopy. By collecting spectral information at each pixel of a two-dimensional (2-D) detector array, HSI generates a three-dimensional (3-D) dataset of spatial and spectral information, known as a hypercube. In its earlier stages, hyperspectral imaging was used as part of an earth remote sensing [11] system. This system employed a sensor which had ability to provide massive quantities of data being scanned.

Medical hyperspectral imaging (MHSI) has been explored for use in some surgeries, such as mastectomy, gall bladder surgery, cholecystectomy, nephrectomy, renal surgery, abdominal surgery, and intestinal surgery [12]. Spectral retina imaging was developed [13] by modifying a fundus camera with spectral imaging device, by integrating a liquid crystal tunable filter (LCTF) and a charge-coupled device (CCD). Wavelengths between 500 and $650 \mathrm{~nm}$ were used to obtain retinal vasculature images to analyse arterioles and venules change.

More recently, a mobile system called PEEK has been [15] developed and implemented [16] (Portable Eye Examination Kit). The mobility and portability of PEEK is its main advantage, and has pioneered its use in Kenyan rural communities to aid detection of eye impairments. The success of PEEK has called for further efforts to identify innovative ways to simplify and advance clinical diagnostic devices.

The principal hardware used to generate hyperspectral images to obtain spectral and spatial information is known as the hyperspectral camera $[14,1]$. This is combined with a light source and a separate processing device to aid massive data storage. There are three types of device configurations which can be used for spatial data collection known as plane, line, and point scanning. Fig. 2 shows the configuration of a line scan system also known as the pushbroom technique. In order to obtain a contiguous image either the subject or the camera must be moved. The movement between the array detector and the frame acquisition rate must synchronized [1].

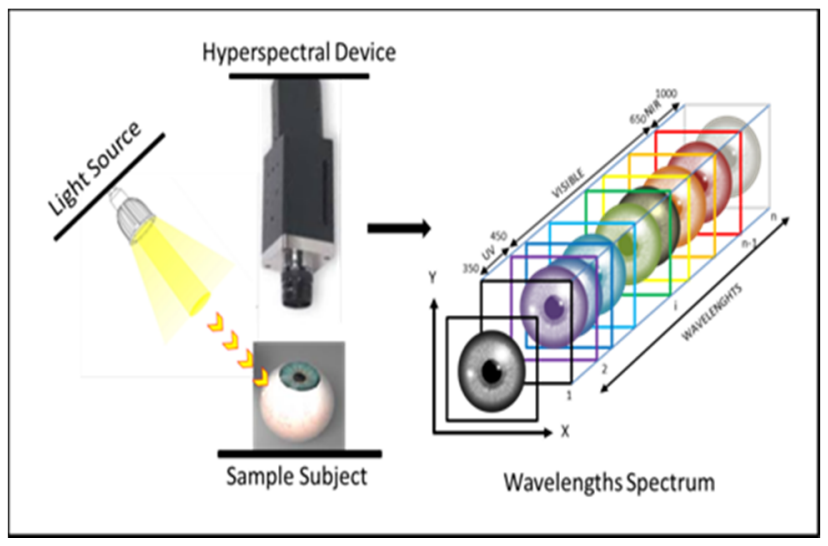

Figure 2.Pushbroom hyperspectral imaging.

\section{EXPERIMENTAL METHOD}

To our knowledge no hyperspectral image database of cornea epithelium exists in the literature to set as a baseline reflectance pattern. The hyperspectral system we used consist of a hyperspectral camera with 400 to $1000 \mathrm{~nm}$ visible wavelengths, two halogen lamps which act as radiation sources located on the left and right side of the frame. The system was calibrated using a white tile to obtain corrected reflectance data. Five porcine eyes (dead tissues) were used and these were defrosted slowly in warm sodium chloride $0.9 \%$. When defrosted, they were fixed on a corkboard to minimise movement. The eyes were moistened with sodium $0.9 \%$ regularly throughout the process to prevent corneal over drying. The eyes were then placed approximately $10 \mathrm{~cm}$ below the camera for optimum image focus.

A pushbroom scanning method was used where the object moved whilst camera remains stationary. The movement of the subject was controlled by an $\mathrm{x}-\mathrm{y}$ axis linear motor where the speed was adjusted to scan and capture images with 1200 to $1300 \times 804 \times 604$ resolution. All eyes were also imaged in standard RGB for comparison.

The Matlab image processing toolbox was utilised to process the images. The header and raw data files were collected for each of these images. All images were read and saved as .mat file format before further processed.

\section{PRELIMINARY ReSUlt AND DisCUSSION}

The images of all the eyes were processed and Eye 1(Fig.3 (a)) was selected to best represent the data as it had areas of both injured and intact epithelium on its surface.

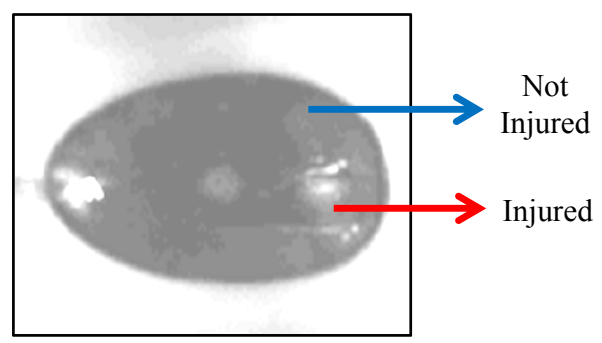

Figure 3(a). Eye 1, Pig's eye with normal and injured cornea. 
Eye 2 (Fig.3(b)) was chosen as a reference subject as it has no identifiable surface injury. The same reflectance spectrum analysis was performed for both eyes for comparison.

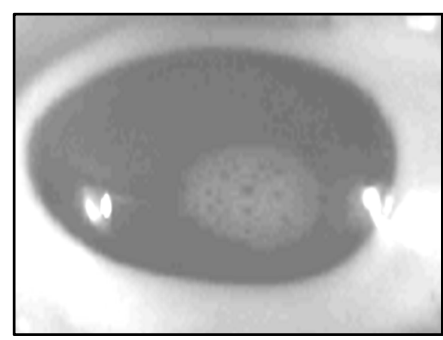

Figure 3(b). Eye 2, Pig's eye with normal cornea.

For Eye 1, the injured surface was determined by slicing the images into area of interest. Fig.4(a),(c),(e),(g) show the gray image at band 518,698,758, and 818 respectively. Images at longer wavelengths reveal distinct shapes in regular arrangements, and is likely to represent individual cells although this require further study for confirmation. While Fig.4(b),(d),(f),(h) show the structure in hsv colour image.

Fig.5(a) shows random placement of 20 pixels on both the injured area (labelled as 1) and the uninjured area (labelled as 2 ), on Eye 1. Information from the pixel points from both areas were averaged and plotted as depicted in Fig.5 (b).

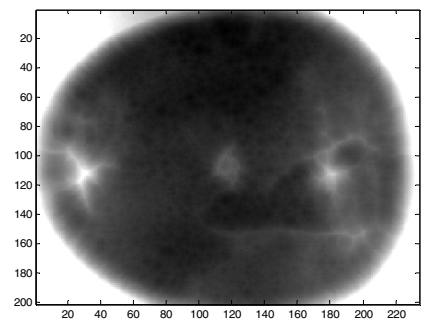

(a)

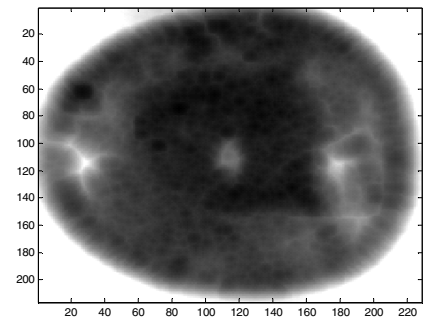

(c)

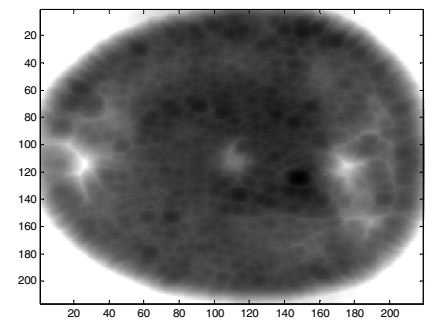

(e)

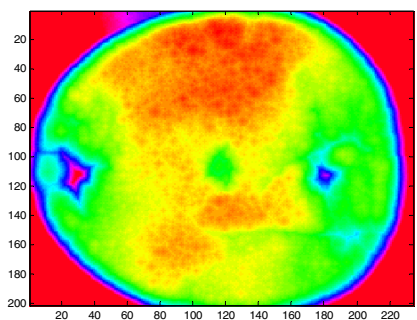

(b)

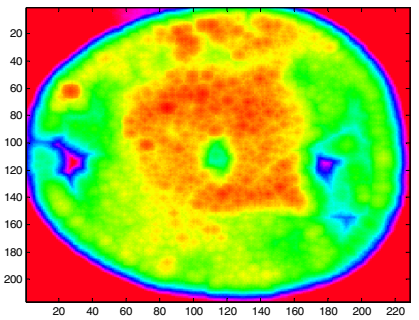

(d)

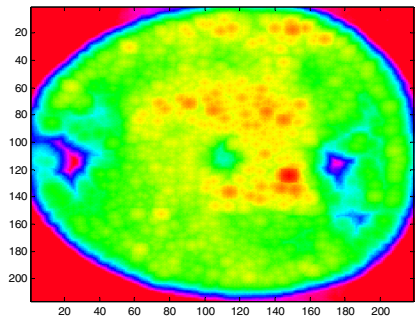

(f)

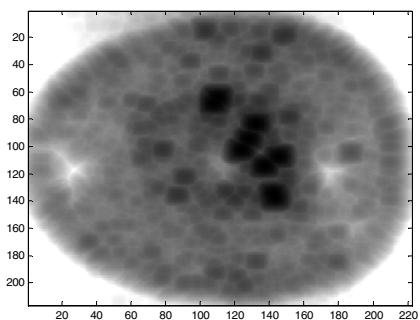

(g)

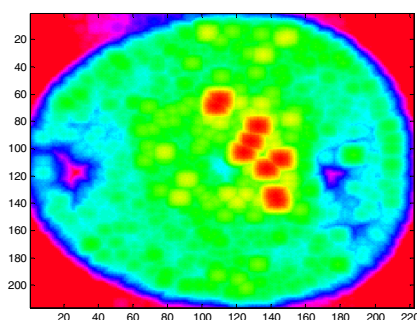

(h)
Figure 4. Image sliced at band 518,698,758, and 818nm; first column (a),(c),(e),(g) represent gray scale images;second column (b),(d),(f),(h) represent hsv color images.

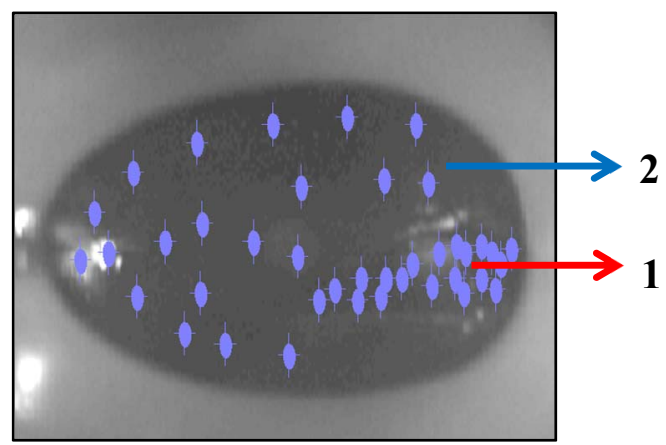

(a)

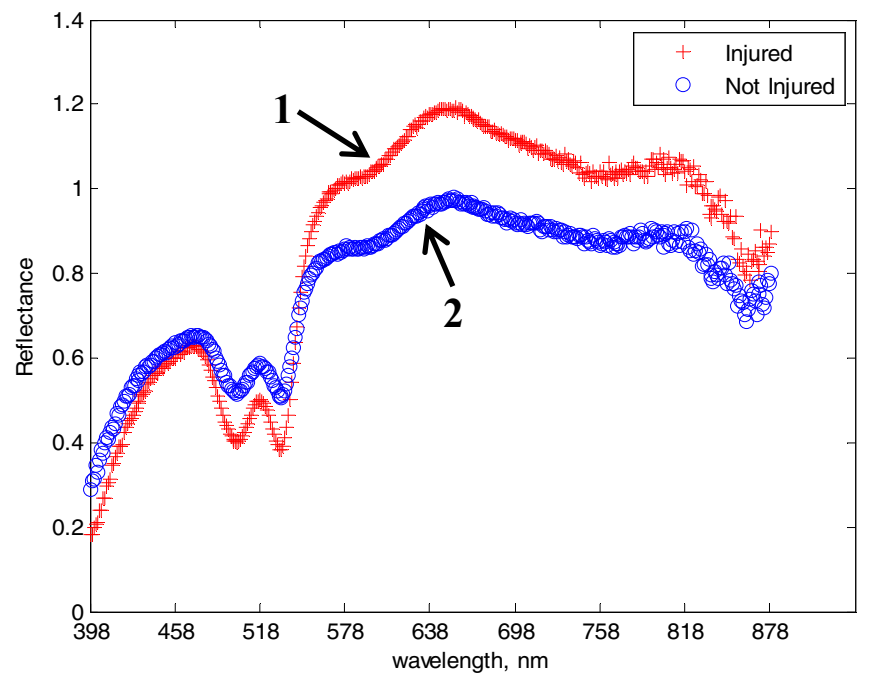

(b)

Figure 5.Eye1, (a) 1- Injured surface, 2- Non injured surface, (b) Reflectance vs wavelength of normal and injured surface.

Fig.5 (b) compares reflectance spectrum from both areas, and shows similar initial outline, but with a distinct separation in reflectance values from wavelength 578 to $818 \mathrm{~nm}$. The corneal base tissue properties are likely to account for the similar spectrum outline, although further work will be required to confirm this. 
In contrast, when pixel points were placed and distributed in identical areas on Eye 2 in Fig. 6(a), there is no gap difference in reflectance between both surfaces, from wavelength 578 to 818 nm (Fig.6 (b)).

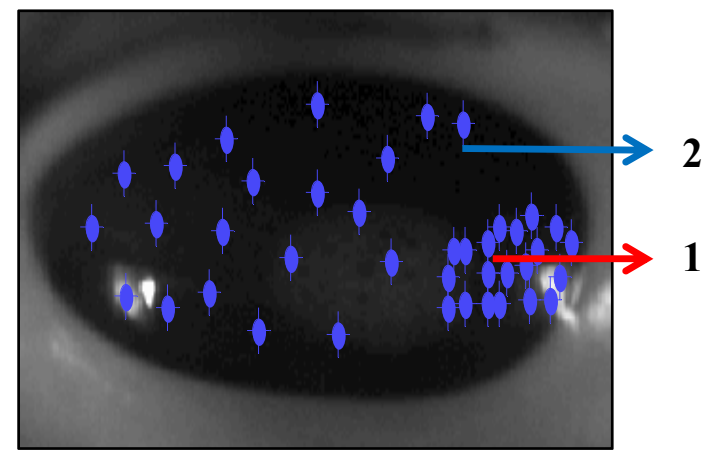

(a)

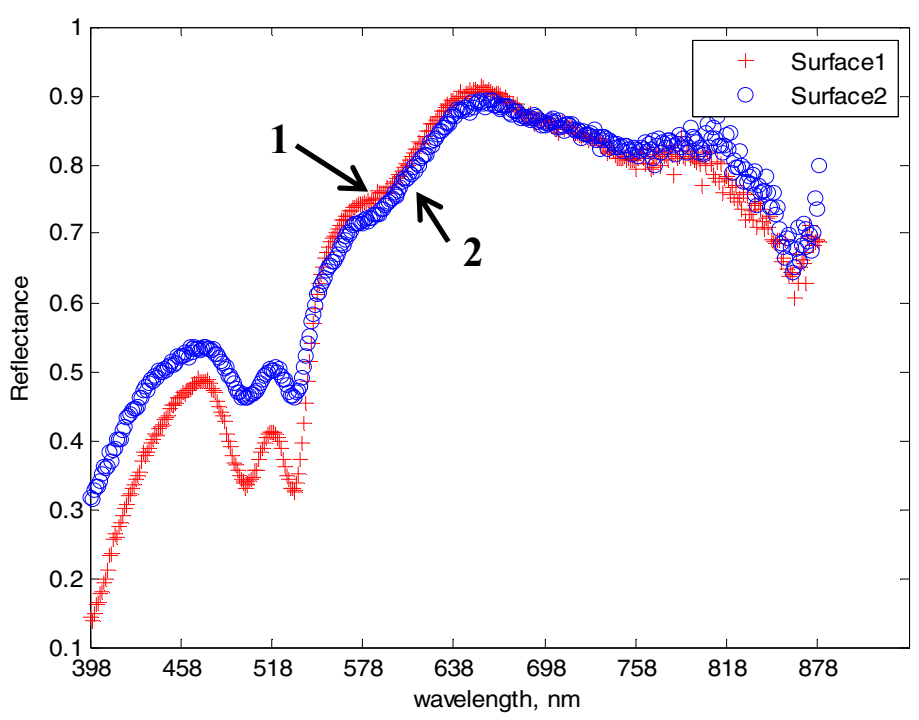

(b)

Figure 6.Eye2, (a) Surface1 \& Surface2 were normal, (b) Reflectance vs wavelength of two normal surfaces.

\section{CONCLUSION AND FURTHER WORKS}

Our analysis was able to demonstrate a gap in the reflectance spectrum between the uninjured and injured part of a porcine's cornea, suggesting it could be of use in assessment of corneal tissue integrity.
Further image processing with grayscale slices reveal distinct tissue properties at varying wavelengths suggesting a novel role for hyperspectral image technology in the diagnostics of corneal tissues, alongside traditional methods such as microscopy. These findings support our proposition for the role of hyperspectral imaging in aiding the development of innovative, mobile devices.

\section{REFERENCES}

[1] Q. Li, X. He, Y. Wang, H. Liu, D. Xu, and F. Guo, "Review of spectral imaging technology in biomedical engineering: achievements and challenges.," J. Biomed. Opt., vol. 18, no. 10, p. $100901,2013$.

[2] T. Jonas, S. F. Memorial, and C. H. Dohlman, "The function of the corneal epithelium in health and disease," Invest. Ophthalmol., vol. 10, no. 6, pp. 383-407, 1971.

[3] R. P. Kirwan, Y. Zheng, A. Tey, D. Anijeet, H. Sueke, and S. B. Kaye, "Quantifying Changes in Corneal Neovascularization Using Fluorescein and Indocyanine Green Angiography," Am. J. Ophthalmol., vol. 154, no. 5, pp. 850-858.e2, 2012.

[4] J. V. Jester, H. F. Li, W. M. Petroll, R. D. Parker, H. D. Cavanagh, G. J. Carr, B. Smith, and J. K. Maurer, "Area and depth of surfactant-induced corneal injury correlates with cell death," Investig. Ophthalmol. Vis. Sci., vol. 39, no. 6, pp. 922-936, 1998.

[5] B. J. Kaluzny, J. J. Kałuzny, A. Szkulmowska, I. Gorczyńska, M. Szkulmowski, T. Bajraszewski, M. Wojtkowski, and P. Targowski, "Spectral optical coherence tomography: a novel technique for cornea imaging.," Cornea, vol. 25, no. 8, pp. 960-965, 2006.

[6] I. Grulkowski, M. Gora, M. Szkulmowski, I. Gorczynska, D. Szlag, S. Marcos, A. Kowalczyk, and M. Wojtkowski, "Anterior segment imaging with Spectral OCT system using a high-speed CMOS camera.," Opt. Express, vol. 17, no. 6, pp. 4842-4858, 2009.

[7] P. B. Morgan and C. Maldonado-Codina, "Corneal staining: Do we really understand what we are seeing?," Contact Lens Anterior Eye, vol. 32 , no. 2 , pp. $48-54,2009$.

[8] J. W. Ruberti, A. S. Roy, and C. J. Roberts, "Corneal biomechanics and biomaterials.," Annu. Rev. Biomed. Eng., vol. 13, pp. 269-295, 2011.

[9] C. Steele, "Corneal wound healing: a review," Optom. Today, pp. 28-32, 1999.

[10] N. Efron, "Contact lens complications quick-find index," in Contact Lens Complications, 2012, p. 350.

[11] a F. Goetz, G. Vane, J. E. Solomon, and B. N. Rock, "Imaging spectrometry for Earth remote sensing.," Science, vol. 228, no. 4704, pp. 1147-1153, 1985

[12] G. Lu and B. Fei, "Medical hyperspectral imaging: a review," $J$. Biomed. Opt., vol. 19, no. 1, p. 10901, 2014.

[13] D. J. Mordant, I. Al-Abboud, G. Muyo, a Gorman, a Sallam, P. Ritchie, a R. Harvey, and a I. McNaught, "Spectral imaging of the retina.," Eye (Lond)., vol. 25, no. 3, pp. 309-320, 2011.

[14] P. L. M. Geladi, H. F. Grahn, and J. E. Burger, "Multivariate Images, Hyperspectral Imaging: Background and Equipment," Tech. Appl. Hyperspectral Image Anal., pp. 1-15, 2007.

[15] F. Lamonaca, G. Polimeni, K. Barbé, and D. Grimaldi, "Health parameters monitoring by smartphone for quality of life improvement," Measurement, 2015.

[16] "Rupert Bourne.," p. 2014, 2014. 